\title{
Investigation of the possibilities for energy economy in continuous dryers through reduction of the drying time
}

\author{
Nina Penkova ${ }^{1, *}$, and Boian Mladenov ${ }^{1}$ \\ ${ }^{1}$ University of Chemical Technology and Metallurgy, Department of Silicate Technology, Blvd. Sv. \\ Kliment Ohridski 8, 1756 Sofia, Bulgaria
}

\begin{abstract}
The possibility for a reduction of the time duration of an industrial drying of ceramic bricks are analysed based on numerical simulation of the transient coupled mass transfer and mechanical processes in the material. The differences between the local mechanical stresses and strength in the dried bodies are computed and used as a criterion for this possibility.

Ways for reorganization of the existing process parameters in the dryers to achieve the corrected drying kinetic curve are discussed. The expected effects on the efficiency of the drying installation are evaluated via material and thermal balance.
\end{abstract}

\section{Introduction}

The drying processes in the ceramic manufacturing are energy intensive and complicated for maintenance [1]. Complex heat transfer, mass transfer and mechanical processes proceed in the so-called green bodies during the different periods of drying [2, 3]. The surface temperature of the articles is changed to the wet-bulb temperature of the drying gas and the evaporated amount of water is relatively small during the Zero period of drying (ZPD). Liquid water evaporation at relatively constant surface temperature, equal to the wet bulb temperature, plastic deformations and shrinkage proceed at the constant-rate drying period (CRDP). The shrinkage stops at the end of that period at the critical moisture content. The surface temperature increases to the drying media temperature and the moisture is changed with decreasing rate at the falling-rate period of drying (FRPD). The proper organisation of the drying regime is important for the energy efficiency of the drying installations and the quality of the products. The drying mode at CRDP has to be "soft" -it has to proceed with enough low rate to prevent failure of the products because of non-uniform moisture content and shrinkage in the green bodies. Higher moisture gradients and non-uniform shrinkage have to be carefully prevented especially at green bodies with complex geometries. The next period (FRDP) can proceed at higher drying rate as the mechanical deformations of the articles are insignificant.

\footnotetext{
*Corresponding author: nina@uctm.edu
} 
The mathematical modelling of the coupled transport phenomena and mechanical processes, based on experimentally obtained data for the material properties of the dried material are appropriate and effective way to obtain detail information about the above processes in order to organise and control proper drying regimes [4, 5].

Mathematical models were developed and numerical simulations of coupled mass transfer and mechanical processes in building bricks at their drying in continuous tunnel dryers were implemented via finite element method [6-8]. The models were validated and calibrated comparing the computed and maintained moisture changes and sizes reductions of the articles [8]. They are used in the present study to check the possibility for a reduction of the drying time and the subsequent energy savings.

\section{Decreasing of the drying time: activities and modeling}

\subsection{Reorganisation of the drying regime}

The drying installation is consisted of continuous drying channels and generators of hot drying gas mixture (fig. 1). Detail information about the installation is not possible because of confidential rules. The drying gas is fed through multiple inlets along the channel. An automation system controls the gas temperature and relative humidity along the channel by varying the mass flows on the different inlets and respectively the fuel and airflows at the generator inlets. The moisture content of the gases $d$ and the drying potential and are presented on figure 2:

$$
\varepsilon=T-T_{w b}, \mathrm{~K}
$$

where $T=$ temperature of the drying gas, $\mathrm{K} ; T_{w b}=$ wet-bulb temperature of the drying gas.

Higher relative humidity and a relatively low temperature are maintained in the first half of the drying tunnel, where CRPD proceeds. These result in soft drying mode due to a low drying potential in that part of the channel.

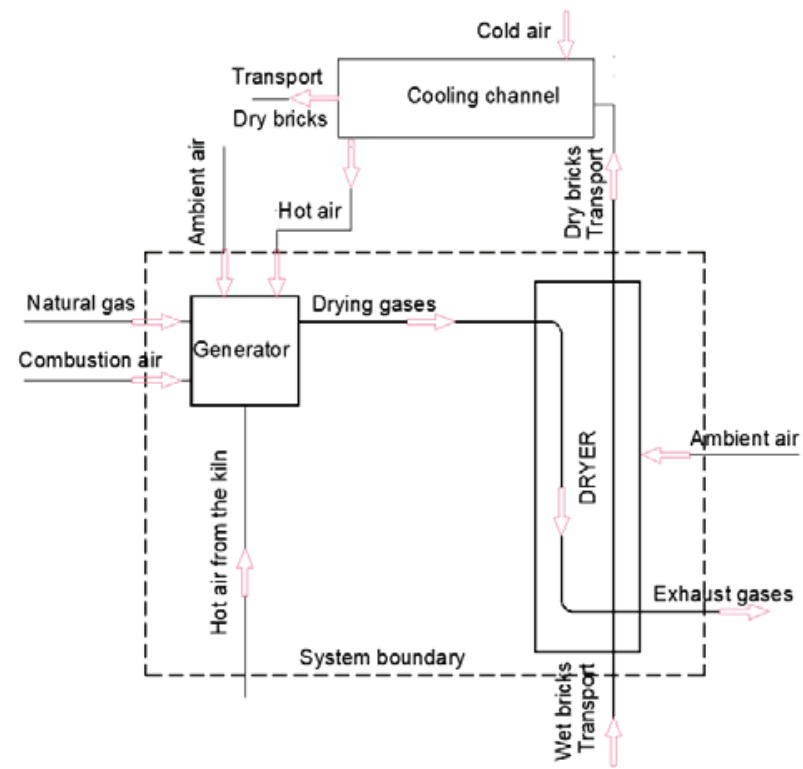

Fig. 1. Common scheme of the drying installation. 


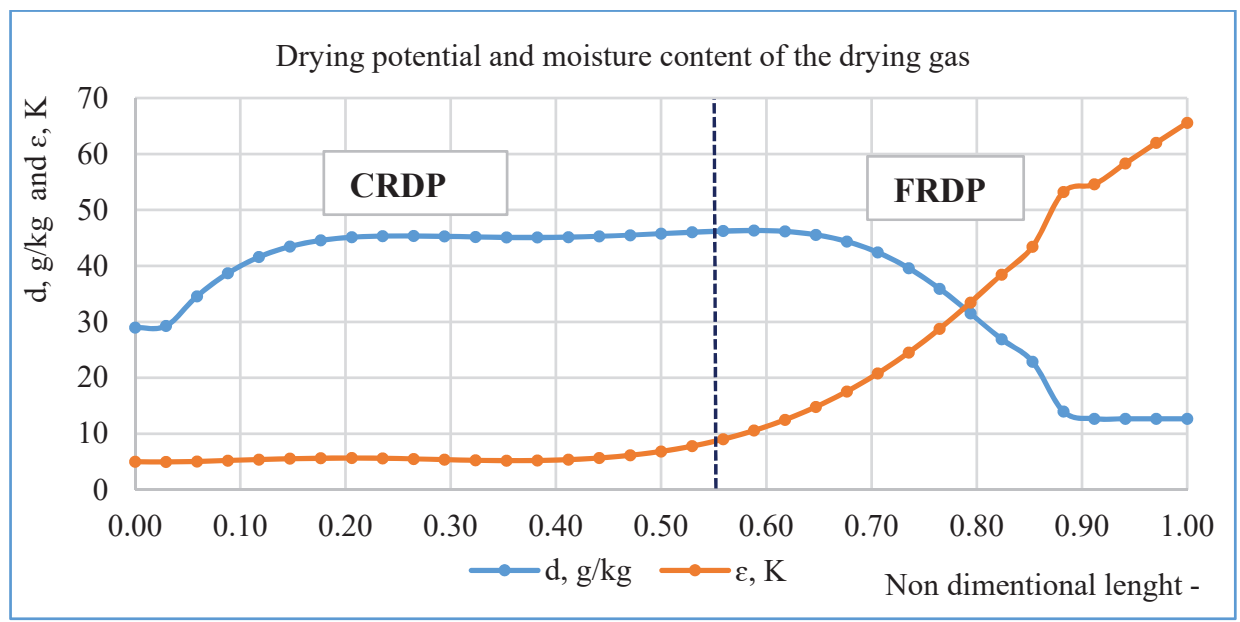

Fig. 2. Change of the drying gas parameters with the length of the dryer.

The energy consumptions at the technological processes are estimated by energy balances [9]. A Sankey diagram (fig. 3) presents such balance of the installation at heat flows, computed at the system boundaries on fig. 1 . A comparatively high energy efficiency of the installation is obvious by the figure: the useful energy for water evaporation is $61 \%$. The low thermal loses contribute to that. The utilized heat flow of the air, cooling the hot ware and transport in the kiln, is $46 \%$ of the input heat flows. That heat recovery leads to a relatively small fuel consumption in the drying installation. The technological efficiency is also high there are not defect and cracks in the dried product at the maintained conditions in the dryers.

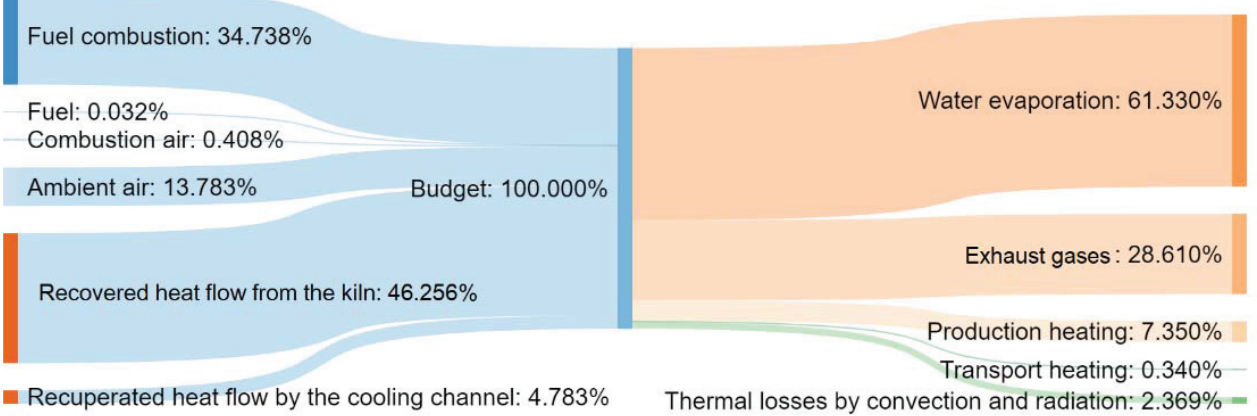

Fig. 3. Energy balance.

Activities and conditions in order to reduce the drying time are accepted:

a reduction of drying time by $7.7 \%$ by shortening the intervals of pushing of the wagons in the drying tunnel by the same percentages;

- a keeping of the present controlled curves of the temperatures and relative humidity along the drying channel length by increasing the mass flows of the drying agent at the inlets of the dryer.

All solid and gas mass flows, and subsequent heat flows in the dryers are increasing whit $8.3 \%$, according to the material and energy balances.

The changes impose also increasing of the speed of the transported bricks and the gas flows in the kiln. They are possible because of the sufficient capacity of the burners and the fans of the kiln and the drying installation. The energy balance of the kiln shows that the mass and heat flow of the hot air from the kiln to the dryers is also increased with $8.3 \%$. 
The thermal loses to the environment are not expected to be changed because of the unchangeable temperatures in the installation. Their keeping at the same level at the increased capacity of the dryers lead to decreasing of the specific energy consumption per kilogram evaporated water. However, the share of heat losses in total energy costs is relatively small (fig. 3) and the energy saving is negligible.

The fuel consumption is increasing with $8 \%$ and the energy consumption per kilogram evaporated water will not be changed significantly. Therefore, the accepted changes are not leading to essential energy efficiency. A positive result is the increase of the capacity of the factory.

\subsection{Modelling and numerical simulation of the mass transfer and stress-strain behaviour in the green body at the forced drying}

The conceptions for modeling, mathematical models and the stages of their calibrations and validations are given in [6 - 8]. The numerical simulations of the structural-diffusion processes are implemented via finite element technics for coupled thermo-mechanical analysis of ANSYS Mechanical APDL [10], based on the analogy between the mass and heat transfer. The field of the moisture content in the green body is computed by:

$$
\rho_{d} c \frac{\partial W}{\partial \tau}=\left[K_{\text {art }}\right] \nabla^{2} W
$$

where $W=$ moisture content of the dried material, referred to the dry mass, $\% ; K_{a r t}=$ artificial coefficient of thermal conductivity, $\mathrm{Wm}^{-1} \mathrm{~K}^{-1} ; \rho_{d}=$ density of the dry mass, $\mathrm{kgm}^{-3} ; c$ $=$ specific heat capacity, $\mathrm{Jkg}^{-1} \mathrm{~K}^{-1}$.

The artificial coefficient of thermal conductivity is computed by the effective coefficient of water diffusion $D_{e f}\left[\mathrm{~m}^{2} \mathrm{~s}^{-1}\right]$, obtained experimentally:

$$
K_{\text {art }}=D_{e f} \rho_{d} c
$$

The boundary conditions include nonlinear mass flux on the mass exchanging boundaries, expressed by an artificial heat flux at the analogy between the heat and mass transfer:

$$
\begin{aligned}
& \left.K_{\text {art }} \frac{\partial W}{\partial n}\right|_{S}=\dot{q}_{a r t} \\
& \dot{q}_{\text {art }}=\left\{\begin{array}{c}
-100 c \dot{q}_{m}=\text { constant } \text { at } W \geq W_{c} \quad(\text { ZPD and } C R P D) \\
100 c \dot{q}_{m}\left(\frac{W_{e}-W}{W_{c}-W_{e q}}\right)^{1 / W} \quad \text { at } 1 \leq W<W_{c} \quad(C R P D) \\
100 c \dot{q}_{m} \frac{W_{e q}-W}{W_{c}-W_{e q}} \quad \text { at }-1<W<1 \quad(C R P D)
\end{array}\right.
\end{aligned}
$$

where $\dot{q}_{m}=$ mass flux of the water, evaporated at the boundary at CRDP, $\mathrm{kgm}^{-2} \mathrm{~s}^{-1} ; W_{c}$ and $W_{e q}$ are the critical and the equilibrium moisture content of the material, obtained experimentally, $\%, \dot{q}_{\text {art }}=$ artificial heat flux, $\mathrm{Wm}^{-2}$.

A relation for Nusselt number as function of Reynolds and Guhman numbers is used to compute the mass flux [6 - 8]. Guhman number is non-dimensional drying potential; it is not changed at the kept temperature and moistures of the gas flows along the channel. Reynolds numbers is increased because of the increased velocities of the gases.

The change of the artificial heat fluxes whit the time before and after the accepted changes is presented on fig. 4 . 


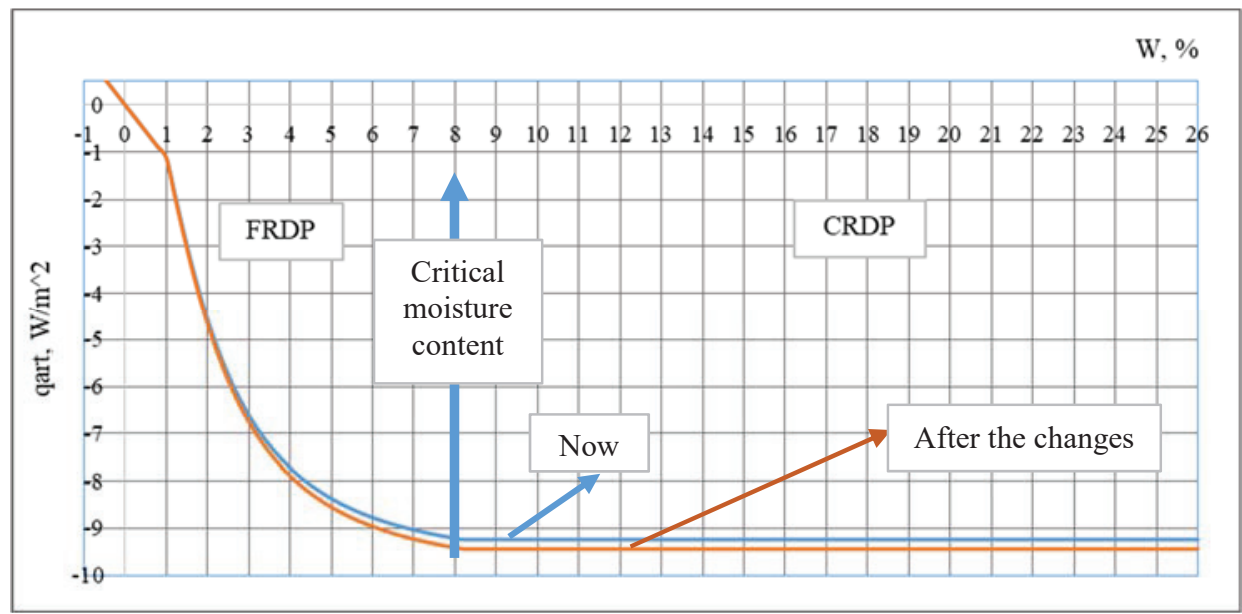

Fig. 4. Artificial heat flux variation with moisture content.

The equation (1) is solved coupled with the stress-strain relationship to obtain the stresses and deformations in the green body:

$$
\{\sigma\}=[D]\{\varepsilon\}
$$

where: $\{\sigma\}=$ stress vector; $\{\varepsilon\}$ - elastic strain vector; $[D]$ - elastic stiffness matrix, formed by module of elasticity $E$ and Poisson ratio $v$.

In direct coupled structural-diffusion analyses the total strain is formed of elastic $\left\{\varepsilon^{e l}\right\}$ and diffusion $\left\{\varepsilon^{d}\right\}$ parts, respectively:

$$
\{\varepsilon\}=\left\{\varepsilon^{e l}\right\}+\left\{\varepsilon^{d}\right\}=[D]^{-1}\{\sigma\}+\{\beta\} \Delta C
$$

where: $\{\beta\}=$ vector of coefficient of diffusion expansion, $\mathrm{m}^{3} \mathrm{~kg}^{-1} ; \Delta \mathrm{C}=$ water concentration change according reference value $C_{\text {ref, }} \mathrm{kgm}^{-3}$ :

$$
\Delta \mathrm{C}=\mathrm{C}-C_{\text {ref }}
$$

The modulus of elasticity $E$, Poisson ratio, coefficient of diffusion expansion $\beta$ and $D_{e f}$ are obtained experimentally and used in the models as functions of moisture content $[6,9]$.

The diffusion strain can be computed as "thermal" strain in equation (7):

$$
\varepsilon^{d}=\beta\left(C-C_{\text {ref }}\right) \rightarrow \varepsilon^{t h}=\alpha\left(T-T_{\text {ref }}\right)
$$

where $\alpha=$ instantaneous coefficient of thermal expansion, $\mathrm{K}^{-1}$. It is accepted equal to $\beta$ at the using of analogy between the structural -diffusion and structural - thermal analysis:

$$
\beta=\frac{\Delta l}{l\left(W_{i n}-W_{c}\right)}
$$

where $W_{\text {in }}$ is the initial moisture content of the material, $\%$.

The boundary conditions in the models of the mechanical processes include zero normal displacements at the contact faces with the transport and the symmetry boundaries (if there exists any). The gravity has to be taken into account to model the plastic deformations. 


\section{Results and discussion}

Numerical simulation of the transient fields of moisture content, stresses and strain are implemented at the corrected parameters of the drying regime for one of the most produced articles. The models are solved numerically for a discretized domain of the body by finite elements Solid 5, suitable for a direct coupled-field analysis [10]. A symmetry plane divides the geometrical model. It is accepted as adiabatic and non mass exchanging. The mass flux at the contact surfaces between the body and the transport is reduced with $80 \%$ according to the arrangement of the production on the wagons in the dryer.

The computed fields at a moment of the process with relatively high stresses in the article are presented on the figures bellow. The moisture concentration is higher in the zones near the contact with the transport (fig. 5). These differences cause non-uniform shrinkage (fig. 6) and subsequent stresses in the ceramic domain. The positive values of the first principal stress on figure 7 (indicative for the ceramic) correspond to the tensile stresses, the negative are the compressive ones. The maximal stresses are established in the zones with the maximal moisture gradients.

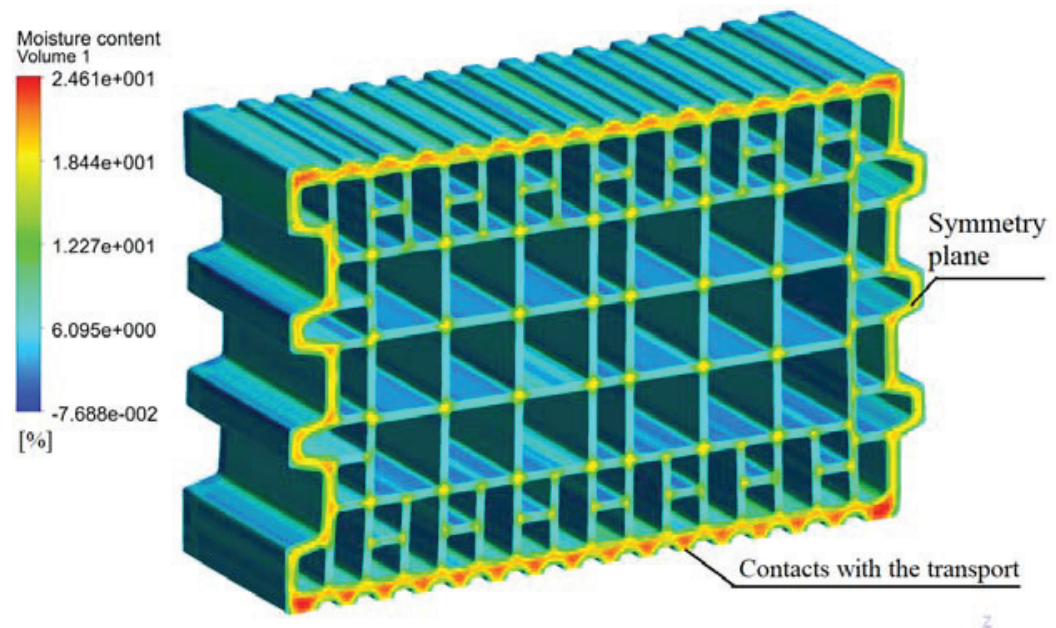

Fig. 5. Moisture content at a moment, corresponding to $44 \%$ of the drying time.

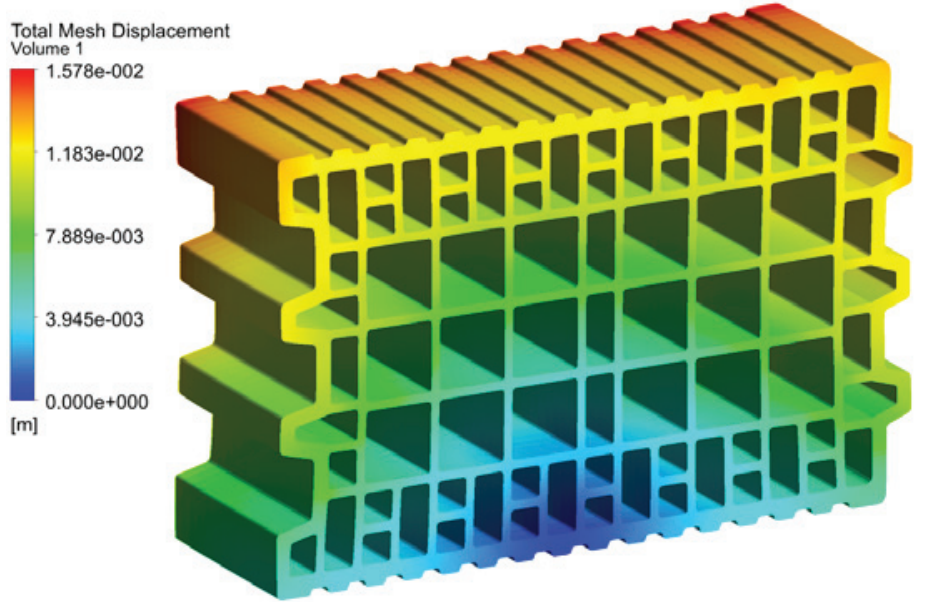

Fig. 6. Total displacements at a moment, corresponding to $44 \%$ of the drying time. 


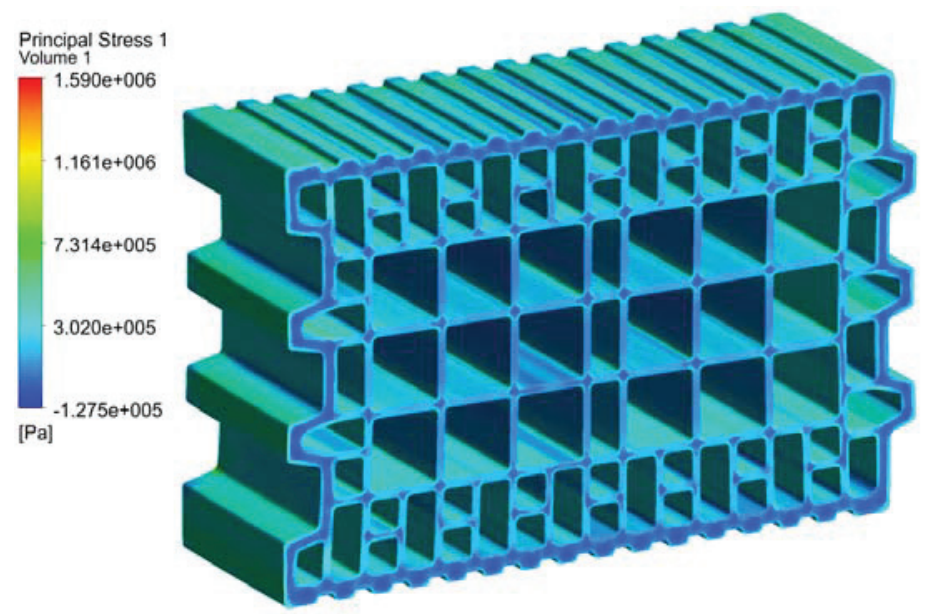

Fig. 7. First principal stress at a moment, corresponding to $44 \%$ of the drying time.

Additionally the modulus of rupture $F_{c f}$ (used as tensile strength for the ceramics) and the compressive strength $F_{c f}$ of the materials are computed for all nodes of the mesh at the nodal moisture contents using relations, obtained via experimentally tests [6].

$$
\begin{gathered}
F_{c f}=35.7 W^{4}-2926.4 W^{3}+84641 W^{2}-1039763.8 W+6040142.3, \mathrm{~Pa} \\
F_{c}=836.2 W^{2}-40704.8 W+549615 \times 10^{-6}, \mathrm{~Pa}
\end{gathered}
$$

The differences between the modulus of rupture and the tensile strength are presented on fig. 8. The difference between the compressive strength and the absolute values of the compressive stresses are obvious from the next figure. The positive values of the figures show that the stresses are lower than the corresponding strengths, with the exception of the compressive stresses at several nodes of the mesh, which can be neglected. Such positive results are established for all moments of the process. Therefore, cracks and defects on the articles are not expected at the suggested time reduction of the process.

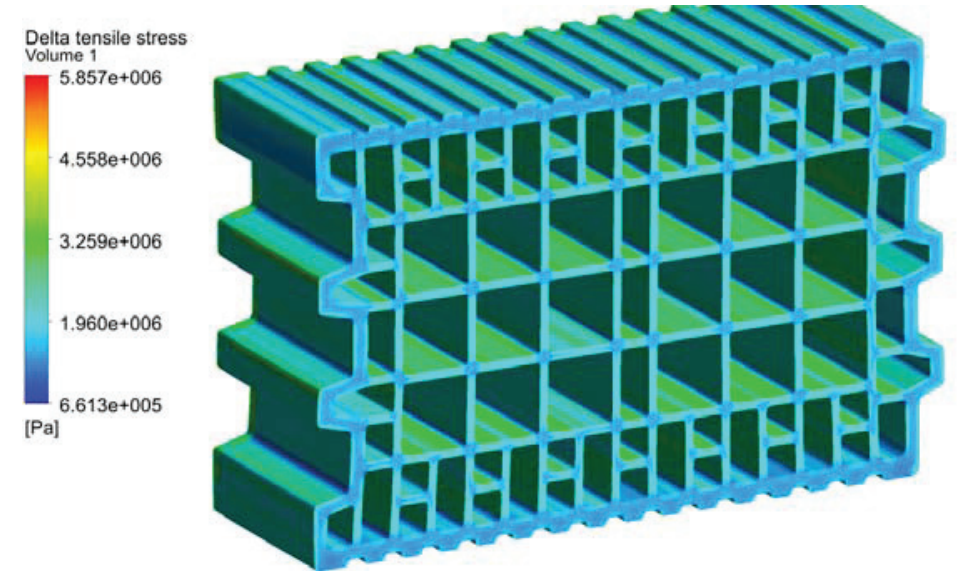

Fig. 8. Differences between the modulus of ruptures and tensile stresses at a moment, corresponding to $44 \%$ of the drying time. 


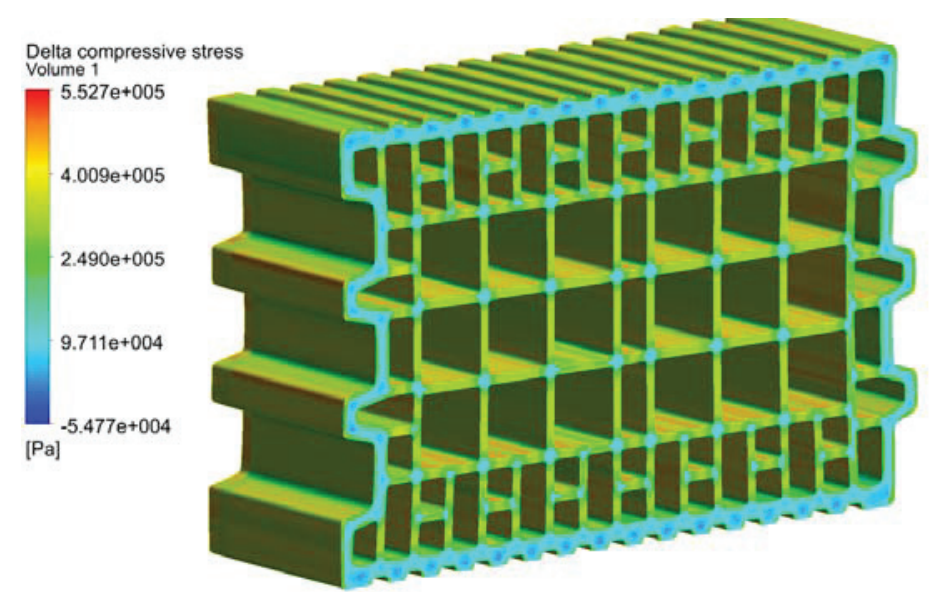

Fig. 9. Differences between the compressive strengths and stresses at $44 \%$ of the drying time.

\section{Conclusions}

The proposed reduction of the drying time is possible at the investigated drying installation: the capacity of the equipment is enough and the forced drying is not dangerous for the quality of the production. However, an improvement of the energy efficiency and reduction of the fuel consumption are not expected. The changes can be implemented if there is a necessity for increasing of the productivity of the enterprise.

The developed algorithm for modeling and numerical simulation of the mass transfer and mechanical processes in the dried materials is suitable for establishment of the limit of reduction of the drying time at keeping the requested quality of the products.

\section{References}

1. European commission, Reference document on best available techniques in the ceramic manufacturing industry (2008)

2. S. Nevenkin, Drying and drying techniques (in Bulgarian), Technika, Sofia (1985)

3. A.V. Lykov, Theory of Drying (in Russian), Moscow (1968)

4. S. Miranda, L. Patruno, M. Ricci, R. Saponelli, F. Ubertini F, Journal of Materials Processing Technology 215, 309-319 (2015)

5. S. Oummadi, Drying behavior of ceramic green bodies: experimental characterization and numerical modeling, Thesis, Université de Limoges (2019)

6. N. Penkova, B. Mladenov, K. Krumov, IOP Conf. Series: Materials Science and Engineering 595, (2019)

7. N. Penkova, B, Mladenov, B. Zlatkov, T. Georgieva, O. Panayotov, Scientific works of University of Food Technology, 66 (1), 151-157 (2019)

8. N. Penkova, B. Mladenov, Proceedings of conference EMF 2019, (in Bulgarian) 64-72 (2019)

9. P. Zlateva, R. Chuturkova, D. Toneva, International Multidisciplinary Scientific GeoConference SGEM, 18 (4.1) 333-340 (2018)

10. ANSYS Release 16 - (C) SAS IP, Inc. (2016) 